\title{
REVIEW
}

\section{Microdissection testicular sperm extraction: an update}

\author{
Ali A Dabaja and Peter N Schlegel
}

Patients with non-obstructive azoospermia (NOA) were once considered to be infertile with few treatment options due to the absence of sperm in the ejaculate. In the last two decades, the advent of intracytoplasmic sperm injection (ICSI), and the application of various testicular sperm retrieval techniques, including fine needle aspiration (FNA), conventional testicular sperm extraction (TESE) and microdissection testicular sperm extraction (micro-TESE) have revolutionized treatment in this group of men. Because most men with NOA will have isolated regions of spermatogenesis within the testis, studies have illustrated that sperm can be retrieved in most men with NOA, including Klinefelter's syndrome (KS), prior history of chemotherapy and cryptorchidism. Micro-TESE, when compared with conventional TESE has a higher sperm retrieval rate (SRR) with fewer postoperative complications and negative effects on testicular function. In this article, we will compare the efficacy of the different procedures of sperm extraction, discuss the medical treatment and the role of testosterone optimization in men with NOA and describe the micro-TESE surgical technique. Furthermore, we will update our overall experience to allow counseling on the prognosis of sperm retrieval for the specific subsets of NOA.

Asian Journal of Andrology (2013) 15, 35-39; doi:10.1038/aja.2012.141; published online 17 December 2012

Keywords: azoospermia; genetics; male infertility; microsurgery; non-obstructive azoospermia; sperm retrieval; testicular sperm extraction

\section{SURGICAL SPERM EXTRACTION}

Azoospermia, defined as the absence of spermatozoa in the ejaculate after assessment of centrifuged semen on at least two occasions, is observed in approximately $1 \%$ of the population and in up to $15 \%$ of infertile men. Non-obstructive azoospermia (NOA), which is diagnosed in about $60 \%$ of azoospermic men, can be detected clinically in men with small-volume testicles, elevated follicle-stimulating hormone (FSH) and of course azoospermia. ${ }^{1}$ Before the advances of intracytoplasmic sperm injection (ICSI) and microsurgery, the options for this group of patients was limited to donor insemination. However, ICSI gave these men the opportunity of using in vitro fertilization (IVF) with sperm retrieved from their own testes. ${ }^{2}$ Although men with NOA have different pathological patterns that can be treated with this approach, the technique of finding the isolated areas of the testicle with sperm production is critical for sperm retrieval and ultimately the success of an IVF cycle. Multiple techniques for sperm retrieval have been described in the literature including fine needle aspiration (FNA), percutaneous testis biopsy, open testicular biopsy or testicular sperm extraction (TESE) and microdissection testicular sperm extraction (microTESE). The main advantages of FNA and percutaneous testis biopsy techniques are their simplicity, low cost and minimal invasiveness. Conversely, it has been shown that a significantly lower sperm retrieval rate (SRR) was obtained when FNA was performed, compared with conventional TESE. ${ }^{3}$ In a conventional TESE procedure, a random incision (or incisions) in the tunica is made and a variable volume of tissue is removed in an attempt to retrieve spermatozoa. ${ }^{4}$ These multiple random tunical incisions or large resection of tissue may result in testicular devascularization and atrophy. Furthermore, postoperative intratesticular bleeding and scar formation lead to disruption of spermatogenesis and hormone production. ${ }^{5}$

Micro-TESE meets the threshold of being an optimal technique for sperm extraction; it is minimally invasive, safe and limits the disruption of testicular function, with a high SRR to allow ICSI. With the guidance of an operating microscope during testicular exploration, the testicular blood supply is visualized and preserved; the seminiferous tubules that are most likely to contain spermatozoa are identified and specifically targeted for extraction and sperm retrieval. In a retrospective comparative study done on a small group of patients, the SRR obtained using micro-TESE was higher than that of conventional TESE in NOA men, specifically in the Sertoli-cell-only histological subtype. ${ }^{6}$ Moreover, micro-TESE has a lower complication rate when compared with other testicular sperm retrieval procedures. ${ }^{7}$ Studies have evaluated the consequence of micro-TESE on the testicles. When comparing the structural changes on ultrasound in patients who underwent conventional TESE with those from the microdissection group, there were fewer acute and chronic changes seen in the microdissection group. Functional evaluation of the testicle post microTESE showed a decrease in the serum testosterone concentrations by $20 \%$ at $3-6$ months followed by rebound to $95 \%$ of the pre-TESE testosterone levels at 18 months postoperatively. ${ }^{5}$ In this article, we will review our technique and update our experience with micro-TESE that was first described by us in $1999 .^{8}$

\section{PREOPERATIVE PREPARATION}

All NOA patients should be evaluated with good history and physical examination, with genetic testing being offered and performed. Y 
chromosome microdeletion testing and karyotype analysis will identify the causes of NOA in up to $17 \%$ of men with NOA. ${ }^{9}$ These tests are of diagnostic and prognostic value. For example, men with AZFc deletion or Klinefelter's syndrome (KS) have a very good prognosis for sperm retrieval. ${ }^{10-12}$ In other cases (e.g., men with complete $\mathrm{AZFa}$ or $\mathrm{AZFb}$ deletions), the chance of sperm retrieval can preoperatively be found to be so low based on genetic testing alone, which limits the option of sperm retrieval and IVF. The results of genetic testing can also be used to counsel couples on the possibility of passing the genetic defect to their offspring and are very important in decision making process in proceeding to IVF.

\section{HORMONAL TREATMENT}

In some instances, medical treatment with hormonal manipulation, or limiting patient exposure to certain toxins such as heat can optimize spermatogenesis prior to sperm retrieval surgery. ${ }^{13} \mathrm{Up}$ to $47 \%$ of men that have impaired spermatogenesis with NOA were found to have hypogonadism. ${ }^{14}$ Spermatogenic failure as consequences of hypogonadism may result from hypothalamic, pituitary or testicular disorders. Effective medical treatment is available for hypogonadism from gonadotropin deficiencies. ${ }^{15}$ However, these patients represent a small proportion of men with NOA. The more common cause of NOA is primary testicular failure that is often characterized by borderline to low serum testosterone levels, relatively elevated circulating estradiol, as well as increased serum FSH levels. It is presumed that most men with low serum testosterone levels will also have low intratesticular testosterone, which is important for spermatogenesis. It is controversial whether low serum testosterone level predicts the success of microTESE, because the serum testosterone is indicative of the testicular function as a whole and may not reflect focal areas of spermatogenesis that are found during micro-TESE. Medical therapies that increase serum testosterone can possibly increase intratesticular testosterone levels as well. The NOA men with a baseline serum testosterone greater than $300 \mathrm{ng} \mathrm{dl}^{-1}$ are unlikely to benefit from hormonal therapy. ${ }^{16} \mathrm{In}$ men who have low serum testosterone levels and relatively elevated estradiol, the use of an aromatase inhibitor (e.g., Anastrozole $1 \mathrm{mg}$ po $\mathrm{qd}$ ) to enhance the testosterone levels is a rational approach that could improve sperm production. ${ }^{12,17,18}$ However, if estradiol levels are appropriate $(\mathrm{T} / \mathrm{E}$ ratio $>10)$, clomiphene citrate $\left(25 \mathrm{mg} \mathrm{day}^{-1}\right)$ or tamoxifen $\left(10 \mathrm{mg} \mathrm{day}^{-1}\right)$ is an appropriate initial therapy. In case the patients do not respond with an increase in testosterone, human chorionic gonadotrophin injections may be added. ${ }^{18}$

\section{HORMONAL TREATMENT IN KS}

Baseline testicular volume, testosterone levels and response to a human chorionic gonadotrophin test have been proposed as preoperative parameters that could predict sperm recovery in men with KS. ${ }^{19}$ Age greater than 35 years at the time of TESE can also affect the SRR for non-mosaic KS patients. ${ }^{20}$ In these men, the SRR was only $50 \% .{ }^{11}$ In our experience, FSH, luteinizing hormone and testicular volume had no predictive value for sperm recovery. ${ }^{11}$ Most KS patients will have low baseline testosterone levels, which is not predictive of sperm retrieval. However, the preoperative testosterone serum level after 3 months of medical treatment is predictive of success at sperm retrieval by micro-TESE in men with KS. Men with low baseline testosterone who responded to medical therapy with a resultant testosterone of greater than $250 \mathrm{ng} \mathrm{dl}^{-1}$ had a $77 \%$ chance of sperm retrieval $v$ s. $55 \%$ for men that did not respond to therapy. ${ }^{11}$ Medical therapy in those who responded appeared to identify a subgroup of KS men with SRR similar to that in men with baseline normal testosterone. The benefit, if any, for patients who responded to medical therapy appeared to occur within 1-2 months of treatment to optimize their testosterone levels.

\section{SURGICAL TECHNIQUE}

Microdissection technique was originally described in $1999^{8}$ as a result of observations made during TESE procedures. To avoid damage to the testicular blood supply, the operating microscope was used to identify subtunical vessels before making biopsy incisions. After observing the testicular parenchyma with high-power magnification, qualitative differences between the seminiferous tubules were observed. ${ }^{8}$ This was further validated using a quantitative analysis that showed that more spermatozoa are present in the larger tubules.

Initially the testis is opened widely in an equatorial plane along the mid portion. This allows wide exposure of seminiferous tubules in a physiological approach that follows intratesticular blood flow (Figure 1). Although a longitudinal incision within the testis is also possible. However, there are limitations to that approach because the blood flow might not be easily identified. A small longitudinal incision limits exposure to the testicular tissue and a longer incision can compromise the testicular blood supply (the primary blood supply to the testis wraps around the posterior and lower pole sections of the testis.). The seminiferous tubules are highly coiled within very fine septae, with centrifugal vessels running in parallel to the tubules and septae. Dissection between the tubules allows access to deeper sections of the seminiferous tubules. It is critical to maintain blood supply along the tubules during the dissection and to avoid separation of tubules from the tunica albuginea. The space between the tubules and tunica has an extensive array of subtunical vessels that are prone to excessive bleeding and very difficult to control. Adverse effects (scarring within the testis after surgery) are most dependent on the amount of bleeding that result after the procedure, mandating meticulous hemostasis. Due to the heterogeneity of sperm production in the testicle, microdissection must permit examination of all seminiferous tubules. Use of the operating microscope at $15 \times$ to $20 \times$ power allows identification of the seminiferous tubules that are most likely to contain sperm. If sperm production is present within a seminiferous tubule, then the tubule appears larger and more opaque (Figure 2). Targeting the larger tubules, improves the yield of sperm retrieval and limits the amount of testicular tissue that needs to be removed by 70 -fold. ${ }^{8,12}$ The sperm retrieval procedure can be tedious and on average require longer operative time than a conventional TESE. ${ }^{21}$ The mean operative time was $1.8 \mathrm{~h}$ (range $0.5-6.6 \mathrm{~h}$ ) for successful micro-TESE and $2.7 \mathrm{~h}$ (range $0.8-7.5 \mathrm{~h}$ ) for attempts in which sperm were not

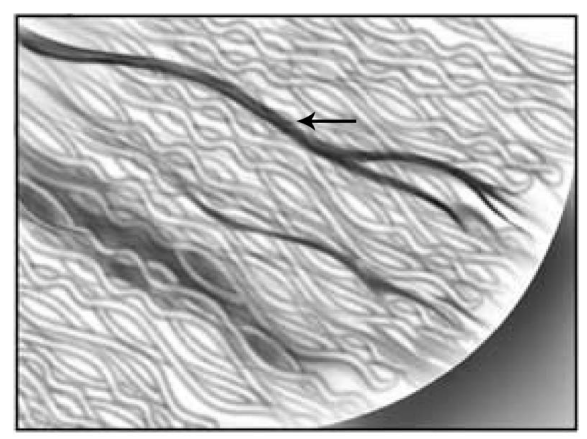

Figure 1 Exposure of seminiferous tubules in a physiologic approach permits the exposure of the intratesticular blood flow and its visualization as illustrated by the arrow. This figure is reproduced with permission from Ramasamy et al. ${ }^{5}$ 


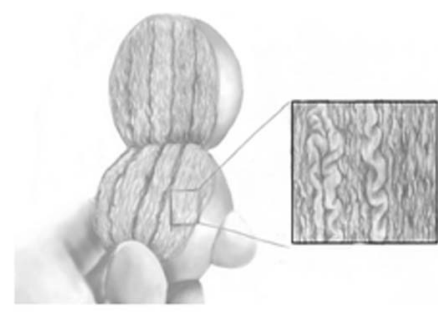

Figure 2 Surgical technique. Use of the operating microscope at 20× power allows identification of the seminiferous tubules that are most likely to contain sperm.

found. Sperm were found within $2 \mathrm{~h}$ in $89 \%$ of the men with a successful Micro-TESE, for procedures that lasted $2 \mathrm{~h}$ to $4 \mathrm{~h}$ success of sperm retrieval was $30 \%$. Furthermore, the success of sperm retrieval was $37 \%$ in procedures that went over $4 \mathrm{~h}^{22}$ There are few studies comparing the cost effectiveness of the different sperm retrieval techniques for NOA. However, studies that examined the economic impact considering both direct and indirect cost of the treatments for varicocele-associated NOA by comparing varicocelectomy to micro-TESE with IVF/ICS, found that micro-TESE is a cost effective procedure. ${ }^{23}$ Surgeon's experience can substantially affect the outcome of micro-TESE, as with many other operations. A considerable number of cases of surgeon experience are necessary to reach a relative plateau level of SRR, and at least 50 cases are needed to pass the steepest portion of the learning curve. Subtle continued increases in SRRs appear to occur as a surgeon exceeds experience with more than 500 micro-TESE procedures. As the number of cases increases, dilated and opaque seminiferous tubules are identified easier and operative time decreases further. These observations reflect the substantial learning curve for microscopic surgery including micro-TESE. ${ }^{24}$

\section{SPERM PROCESSING}

The mechanical processing of testicular tissue samples in the operating room should involve cutting tissue samples and passing the resulting suspension through a fine angiocatheter. ${ }^{25}$ This processing dramatically increases the yield of testicular sperm for intraoperative wet preparation analysis by 300 -fold, facilitating early identification of sperm in the operating room. Each specimen should be sequentially examined for the presence of sperm in the operating room, as the procedure can be terminated once sperm are found. However, sperm are not always successfully identified in the operating room; therefore, a meticulous laboratory-based search of the mechanically processed testicular tissue, including enzymatic treatment, is performed to improve the chances of finding sperm. ${ }^{26}$ The enzymatic treatment has shown a range of results, and some studies found that the chance of finding sperm following chemical digestion when sperm were not initially found is $25 \%-30 \% .{ }^{27}$ Such results reflect incomplete initial evaluation of sperm specimens as a 'wet-prep'. Our laboratories reported a success rate of $7 \%$ when effective mechanical preparation of the removed specimen is followed by a multihour, many-technician search for spermatozoa in an experienced laboratory. ${ }^{28}$ These results are important for surgeons to consider when they counsel patients and their partners in the immediate postoperative period, particularly when no sperm are identified intraoperatively. Sperm from men with NOA may not survive freeze-thaw, so intentional removal of additional testicular tissue may waste the sperm production that is present, and create additional scar tissue within the testis, potentially compromising future attempts at fertility treatment.

\section{VARICOCELE REPAIR}

The indications for varicocelectomy in NOA are controversial. A meta-analysis of uncontrolled trials of varicocele repair in NOA suggested that certain pathological subtypes of NOA can benefit from varicocelectomy prior to attempted sperm retrieval surgery, and motile sperm was reported in $39 \%$ of men who underwent a varicocele repair. ${ }^{29}$ However, these subsets of patients are the same men who are likely to have sperm in the ejaculate despite a history of prior azoospermia. In addition, an extremely low spontaneous pregnancy rate of $6 \%$ was also reported. In patients with a known history of varicocele, the result of the micro-TESE is not affected by whether the varicocele was repaired before the micro-TESE in one report. ${ }^{30}$ However, other studies have suggested a benefit of varicocele repair in enhancing sperm retrieval for men with NOA. Based on our experience, we usually restrict varicocele repair to men who have oligospermia or have several years to benefit from sperm in the ejaculate. The benefits of varicocelectomy, if any, may take 3-6 months or longer to be realized and nearly all men who have varicoceles and NOA will require testicular sperm extraction regardless of whether varicocele repair is done. ${ }^{30}$

\section{PREVIOUS BIOPSY}

The necessity of diagnostic biopsy has been debated in the literature. Testicular biopsy can document normal sperm production, and therefore imply the presence of obstruction in normal testicular size with at least one palpable vas deferens and a normal serum FSH $\left(<7 \mathrm{IU} \mathrm{l}^{-1}\right)$. In some institutions, a diagnostic biopsy is also the treatment for men with NOA. For most men with NOA, a single biopsy is inadequate to retrieve sperm and more extensive testicular evaluation may be needed before spermatozoa are found. Multiple random biopsies will carry the risk of creating intratesticular scar, decreasing androgen levels and future sperm production. ${ }^{31}$ Since a diagnostic testicular biopsy provides such a limited evaluation of testicular function, it cannot determine which men with NOA will have successful sperm retrieval. In patients that undergo one to two biopsies with no spermatozoa identified, the success rate of micro-TESSE is close to $60 \% .^{32}$ Therefore, there is no threshold for the number of negative biopsies that precludes sperm retrieval using micro-TESE.

Some retrospective studies reported that a repeat conventional TESE yields a recovery rate of $78 \%$ in sertoli cell-only syndrome and $70 \%$ in maturation arrest. But, most of these men had sperm recovery on the first TESE. ${ }^{33}$ For men who undergo conventional TESE and fail to have sperm retrieval, a repeat conventional TESE causes further testicular damage with limited success. In case of a failed conventional TESE, a salvage microTESE can be offered and spermatozoa retrieval is possible with an SRR of $45 \%{ }^{34}$ In addition, previous micro-TESE seems to have no or little effect on the success of future microdissection. A repeat micro-TESE in men who underwent one successful microdissection attempt carrys an SRR of $82 \%{ }^{35}$ If a complete micro-TESE procedure is accomplished by examining the deeper tissues of the upper and lower poles of both testes and not just the initially exposed midportion, then we would not repeat this operation. Unfortunately, we have found that some surgeons are reporting having performed a 'micro-TESE' procedure and only dissecting a very limited amount of tissue - that initially exposed during the procedure. Our SRR for repeating previously failed 'micro-TESE' procedures done elsewhere is $10 \%$.

\section{OVERALL RESULTS WITH MICRO-TESE}

In a prospective comparative study, comparing conventional TESE using a single extended incision to TESE through multiple incisions, both groups had very low SRRs of $29.5 \%$ and $26.7 \%$, respectively. ${ }^{36}$ Multiple maneuvers have been tried to improve the overall retrieval 
rates, including performing multiple biopsies or the use of Doppler ultrasound prior to TESE to perform biopsy/aspiration in testicular regions with larger blood vessels. ${ }^{37}$ Systematic reviews of the literature have shown that SRRs with micro-TESE are superior to other surgical approaches for sperm retrieval especially in patients with predominant Sertoli-cell-only, the condition found in most men with NOA. ${ }^{38}$ A comparative study including 116 men found a significantly higher SRR with micro-TESE $47 \%$ vs. $30 \%$ in conventional TESE. ${ }^{39}$ Additional retrospective studies that compared conventional TESE with micro-TESE showed $16.7 \%$ SRR vs. $44.6 \%$ SRR with micro-TESE. Furthermore, micro-TESE appears to be particularly effective in the retrieval of spermatozoa in KS patients. ${ }^{12}$ Encouraging experience has been obtained at Weill Cornell with TESE-ICSI in the past 1414 attempted treatment cycles for couples in whom the man had NOA. The mean age of patients entering treatment was 35.5 years for men and 30.4 years for women. In men, the initial mean serum FSH level was 25.3 $\mathrm{IU}^{-1}$ (normal, 1-8 IU $\mathrm{l}^{-1}$ ), and average testicular volume was $13 \mathrm{ml}$. During the past 1414 attempted TESE-ICSI cycles, sperm were retrieved for injection in $794(56 \%)$ cycles $(52 \%(607 / 1176)$ retrieval rate per patient). For those cycles in which sperm were retrieved, the fertilization rate per injected oocyte was $51 \%$ (4423 of 8705). Our embryo transfer rate was $94 \%$. Clinical pregnancies were established in $48 \%$ of the cycles, and live deliveries occurred in $41 \%$ of couples. Multiple gestations were seen in $10 \%$ of pregnancies.

Micro-TESE combined with ICSI is becoming a treatment of choice for certain subsets of men diagnosed with NOA who want to father children with limited options. In this article we updated our series and discussed four NOA patient subsets: KS, cryptorchidism, prior history of chemotherapy and Y chromosome (AZFc) microdeletions. These groups benefit the most from a micro-TESE since their options to father children are limited. ${ }^{11,12,40,41}$

\section{Klinefelter syndrome}

Successful TESE-ICSI for men with non-mosaic KS with delivery of healthy children was first reported in $1996 .{ }^{42}$ Since then, several groups have published small series on the successful pregnancy outcomes combining these techniques. ${ }^{10}$ The treatment of men with KS has been thought to be limited by the small testicular volume, extensive tubular sclerosis and typically found high FSH. In a report by Schiff et al., ${ }^{43}$ the chance of sperm retrieval for men with KS who underwent micro-TESE was around $72 \%$. The results obtained in this study is likely attributed to the effectiveness of micro-TESE technique at finding small, limited areas of sperm production within the testis. We update here the results for treatment of 127 men with classic and mosiac KS (47,XXY, or mosaic patterns that do not include 46,XY) who underwent attempted sperm retrieval during simultaneous 155 ICSI cycles at our institution. Sperm were found in 65\% (100/155) of the fresh retrieval attempts. Our per-patient success rate of sperm retrieval for these 127 men was $61 \%$ (77/127). The median number of embryo transferred was two and transfer occurred in $83 \%$ of the cases, with clinical pregnancy and fertility of $40 \%$. Forty children born to date with multiple gestation rate of $31 \%$ have been seen in these pregnancies. Results did not differ for mosaic or non-mosaic patients. All children have been healthy $(46, \mathrm{XX}$ girls and 46,XY boys). These findings illustrate the potential for TESEICSI to provide fertility despite underlying genetic abnormalities.

\section{Post-chemotherapy}

Another treated subset of men with NOA is patients with history of chemotherapy; our recommendation is that sperm banking be offered before any chemotherapeutic therapy, even if the chance of azoospermia is thought to be small. Our data demonstrate that many men with long-term azoospermia after chemotherapy can still have their fertility salvaged with the use of assisted reproductive techniques. In a cohort of 93 men with a history of chemotherapy administered for a variety of diagnoses underwent 114 sperm retrieval attempts for persistent NOA. Thirty of the 93 (32\%) patients had also received extragonadal radiation. Sperm were successfully retrieved in $48 \%$ (55/114) of micro-TESE attempts, with clinical pregnancy occurring in $40 \%$ of couples. Per-patient SRR was $42 \%$ (39/93). Men treated for lymphoma had an SRR per cycle of $44 \%$, whereas after treatment for germ cell tumor, the SRR was $70 \%$.

\section{Cryptorchidism}

Several case reports have described successful pregnancies after TESE with ICSI for men with NOA associated with cryptorchidism. ${ }^{40,44}$ While the undescended testicle results in a loss of germ cells, including spermatogonia with subsequent NOA, the treatment with an orchiopexy has multiple risk factors and might cause ischemic insult to the testicle. It is believed that orchiopexy has no benefit for seminiferous tubules that have undergone irreversible degeneration. ${ }^{45}$ However, it serves to preserve the foci of germ cells capable of normal spermatogenesis. In our experience, testicular volume and age at orchiopexy were predictive of TESE success. Patients with successful TESE sperm retrieval had a mean testicular volume of more than $2 \mathrm{ml}(8.4 \mathrm{ml} v s .6 .3 \mathrm{ml})$ greater than those with failed retrieval attempts. Furthermore, in patients with successful retrieval procedures, orchiopexy was performed an average of 10 years earlier than in men with failed retrieval (age 10.5 vs. 21.8 years). ${ }^{40}$ Patients treated with early orchiopexy have greater preservation of testicular tissue and allow for the harvesting and a subsequent successful TESE procedure. In 152 men, 181 micro-TESE procedures were performed. Spermatozoa were successfully retrieved in 116/181 (64\%), pregnancy rate was $50 \%$ and the delivery rate was $38 \%$ with eight spontaneous abortions. In this subgroup, the per-patient SRR was $62 \%(94 / 152)$. In our cohort, 39\% had bilateral cryptorchidism, and four patients with history of other genetic abnormality. The SRR in men with a history of bilateral cryptorchidism was $62 \%$.

\section{AZF deletion}

As presented above, genetic testing for Y chromosome microdeletion is of prognostic significance for micro-TESE procedure. For men with complete deletions of the AZFa, AZFb region or absence of AZFa-c, the seminal phenotype is invariably azoospermic, and the chance of sperm retrieval during TESE is severely impaired as it is very clearly shown in the literature. ${ }^{12}$ Therefore, for men with complete AZFa or $\mathrm{AZFb}$ deletions, we do not recommend proceeding with TESE. However, for cycles involving men with AZFc deletions alone (the only Y-deleted patients who have had sperm in our experience), spermatozoa were successfully retrieved in 39/54 (72\%) micro-TESE procedures. The per-patient retrieval rate was $67 \%(26 / 39)$ and pregnancy occurred in $46 \%$ of the treatment attempts (Table 1). All children

Table 1 SRR and pregnancy rate in microdissection testicular sperm extraction (Micro-TESE)

\begin{tabular}{|c|c|c|c|}
\hline & $\begin{array}{c}\text { SRR per Micro- } \\
\text { TESE cycle }\end{array}$ & SRR per patient & Pregnancy rate \\
\hline Chemotherapy & $48 \%(55 / 114)$ & $42 \%(39 / 93)$ & $40 \%$ \\
\hline $\begin{array}{l}\text { Klinefelter's } \\
\text { syndrome }\end{array}$ & $65 \%(100 / 155)$ & $61 \%(77 / 127)$ & $40 \%$ \\
\hline Cryptorchidism & $64 \%(116 / 181)$ & $62 \%(94 / 152)$ & $50 \%$ \\
\hline AZFc deletion & $72 \%(39 / 54)$ & $67 \%(26 / 39)$ & $46 \%$ \\
\hline Overall experience & $56 \%(794 / 1414)$ & $52 \%(607 / 1176)$ & $48 \%$ \\
\hline
\end{tabular}

Abbreviation: SRR, sperm retrieval rate. 
born were phenotypically normal, but we expect all boys to have deletions involving the AZFc region, with resulting impairments in spermatogenesis.

\section{CONCLUSION}

The seminiferous tubules of patients with NOA are usually heterogeneous in structure and function. Conventional TESE cannot discriminate areas of the testis with sperm production, as it reflects a random sample of testicular tissue, so it may be less successful in NOA patients. MicroTESE with an operative microscope is advantageous because larger, more opaque seminiferous tubules likely containing germ cells with active spermatogenesis can be identified directly. This procedure seems to be the best method for the intraoperative identification of sperm producing tubules, resulting in a high spermatozoa retrieval rate and minimal postoperative complications. A high SRR with micro-ESE is clearly demonstrated in men with KS, cryptorchidism, prior history of chemotherapy and $\mathrm{Y}$ chromosome (AZFc) microdeletions as well as idiopathic NOA. Moreover, in certain conditions, it is possible that the chance of SRR can be maximized by pre-treatment of patients to medically optimized hormonal levels, especially in men with KS.

\section{COMPETING FINANCIAL INTERESTS}

Authors have no competing financial interests.

\section{ACKNOWLEDGMENTS}

Illustration by Vanessa Dudley.

1 Jarow JP, Espeland MA, Lipshultz LI. Evaluation of the azoospermic patient. J Urol 1989; 142: 62-5.

2 Devroey P, Liu J, Nagy Z, Goossens A, Tournaye H et al. Pregnancies after testicular sperm extraction and intracytoplasmic sperm injection in non-obstructive azoospermia. Hum Reprod 1995; 10: 1457-60.

3 Hauser R, Botchan A, Amit A, Ben Yosef D, Gamzu R et al. Multiple testicular sampling in non-obstructive azoospermia_-is it necessary? Hum Reprod 1998; 13: 3081-5.

4 Ezeh UI, Moore HD, Cooke ID. A prospective study of multiple needle biopsies versus a single open biopsy for testicular sperm extraction in men with non-obstructive azoospermia. Hum Reprod 1998; 13: 3075-80.

5 Ramasamy R, Yagan N, Schlegel PN. Structural and functional changes to the testis after conventional versus microdissection testicular sperm extraction. Urology 2005; 65: 1190-4.

6 Okada H, Dobashi M, Yamazaki T, Hara I, Fujisawa M et al. Conventional versus microdissection testicular sperm extraction for nonobstructive azoospermia. J Urol 2002; 168: 1063-7

7 Amer M, Ateyah A, Hany R, Zohdy W. Prospective comparative study between microsurgical and conventional testicular sperm extraction in non-obstructive azoospermia: follow-up by serial ultrasound examinations. Hum Reprod 2000; 15: 653-6.

8 Schlegel PN. Testicular sperm extraction: microdissection improves sperm yield with minimal tissue excision. Hum Reprod 1999; 14: 131-5.

9 Rucker GB, Mielnik A, King P, Goldstein M, Schlegel PN. Preoperative screening for genetic abnormalities in men with nonobstructive azoospermia before testicular sperm extraction. J Urol 1998; 160: 2068-71.

10 Friedler S, Raziel A, Strassburger D, Schachter M, Bern 0 et al. Outcome of ICSI using fresh and cryopreserved-thawed testicular spermatozoa in patients with non-mosaic Klinefelter's syndrome. Hum Reprod 2001; 16: 2616-20.

11 Ramasamy R, Ricci JA, Palermo GD, Gosden LV, Rosenwaks Z et al. Successful fertility treatment for Klinefelter's syndrome. J Urol 2009; 182: 1108-13.

12 Schlegel PN. Nonobstructive azoospermia: a revolutionary surgical approach and results. Semin Reprod Med 2009; 27: 165-70.

13 Jung A, Schuppe HC. Influence of genital heat stress on semen quality in humans. Andrologia 2007; 39: 203-15.

14 Bobjer J, Naumovska M, Giwercman YL, Giwercman A. High prevalence of androgen deficiency and abnormal lipid profile in infertile men with non-obstructive azoospermia. Int J Androl 2012; 35: 688-94.

15 Depenbusch M, von Eckardstein S, Simoni M, Nieschlag E. Maintenance of spermatogenesis in hypogonadotropic hypogonadal men with human chorionic gonadotropin alone. Eur J Endocrinol 2002; 147: 617-24.

16 Reifsnyder JE, Ramasamy R, Husseini J, Schlegel PN. Role of optimizing testosterone before microdissection testicular sperm extraction in men with nonobstructive azoospermia. J Urol 2012; 188: 532-7.
17 Cavallini G, Beretta G, Biagiotti G. Preliminary study of letrozole use for improving spermatogenesis in non-obstructive azoospermia patients with normal serum FSH. Asian J Androl 2011; 13: 895-7.

18 Raman JD, Schlegel PN. Aromatase inhibitors for male infertility. J Urol 2002; 167 624-9.

19 Madgar I, Dor J, Weissenberg R, Raviv G, Menashe Y et al. Prognostic value of the clinical and laboratory evaluation in patients with nonmosaic Klinefelter syndrome who are receiving assisted reproductive therapy. Fertil Steril 2002; 77: 1167-9.

20 Okada H, Goda K, Yamamoto Y, Sofikitis N, Miyagawa I et al. Age as a limiting factor for successful sperm retrieval in patients with nonmosaic Klinefelter's syndrome. Fertil Steril 2005; 84: 1662-4

21 Tsujimura A, Matsumiya K, Miyagawa Y, Tohda A, Miura H et al. Conventional multiple or microdissection testicular sperm extraction: a comparative study. Hum Reprod 2002; 17: 2924-9.

22 Ramasamy R, Fisher ES, Ricci JA, Leung RA, Schlegel PN. Duration of microdissection testicular sperm extraction procedures: relationship to sperm retrieval success. J Urol 2011; 185: 1394-7.

23 Lee R, Li PS, Goldstein M, Schattman G, Schlegel PN. A decision analysis of treatments for nonobstructive azoospermia associated with varicocele. Fertil Steril 2009; 92: 188-96.

24 Ishikawa T, Nose R, Yamaguchi K, Chiba K, Fujisawa M. Learning curves of microdissection testicular sperm extraction for nonobstructive azoospermia. Fertil Steril 2010; 94: 1008-11.

25 Ostad M, Liotta D, Ye Z, Schlegel PN. Testicular sperm extraction for nonobstructive azoospermia: results of a multibiopsy approach with optimized tissue dispersion. Urology 1998; 52: 692-6.

26 Crabbé E, Verheyen G, Silber S, Tournaye H, van de Velde $\mathrm{H}$ et al. Enzymatic digestion of testicular tissue may rescue the intracytoplasmic sperm injection cycle in some patients with non-obstructive azoospermia. Hum Reprod 1998; 13: 2791-6.

27 Aydos K, Demirel LC, Baltaci V, Unlu C. Enzymatic digestion plus mechanical searching improves testicular sperm retrieval in non-obstructive azoospermia cases. Eur J Obstet Gynecol Reprod Biol 2005; 120: 80-6.

28 Ramasamy R, Reifsnyder JE, Bryson C, Zaninovic N, Liotta D et al. Role of tissue digestion and extensive sperm search after microdissection testicular sperm extraction. Fertil Steril 2011; 96: 299-302.

29 Weedin JW, Khera M, Lipshultz LI. Varicocele repair in patients with nonobstructive azoospermia: a meta-analysis. J Urol 2010; 183: 2309-15.

30 Schlegel PN, Kaufmann J. Role of varicocelectomy in men with nonobstructive azoospermia. Fertil Steril 2004; 81: 1585-8.

31 Herwig R, Tosun K, Pinggera GM, Soelder E, Moeller KT et al. Tissue perfusion essential for spermatogenesis and outcome of testicular sperm extraction (TESE) for assisted reproduction. J Assist Reprod Genet 2004; 21 : 175-80.

32 Ramasamy R, Schlegel PN. Microdissection testicular sperm extraction: effect of prior biopsy on success of sperm retrieval. J Urol 2007; 177: 1447-9.

33 Vernaeve V, Verheyen G, Goossens A, van Steirteghem A, Devroey P et al. How successful is repeat testicular sperm extraction in patients with azoospermia? Hum Reprod 2006; 21: 1551-4.

34 Tsujimura A, Miyagawa Y, Takao T, Takada S, Koga M et al. Salvage microdissection testicular sperm extraction after failed conventional testicular sperm extraction in patients with nonobstructive azoospermia. J Urol 2006; 175: 1446-9; discussion 9.

35 Ramasamy R, Ricci JA, Leung RA, Schlegel PN. Successful repeat microdissection testicular sperm extraction in men with nonobstructive azoospermia. J Urol 2011 185: 1027-31.

36 Fahmy IM, Mansour RT, Serour GI, Aboulghar M. Single large testicular biopsy is comparable to multiple biopsies to retrieve spermatozoa in patients with nonobstructive azoospermia. Fertil Steril 2000; 74: S86-7.

37 Tunc L, Alkibay T, Kupeli B, Tokgoz H, Bozkirli I et al. Power Doppler ultrasound mapping in nonobstructive azoospermic patients prior to testicular sperm extraction. Arch Androl 2005; 51: 277-83.

38 Donoso $\mathrm{P}$, Tournaye $\mathrm{H}$, Devroey $\mathrm{P}$. Which is the best sperm retrieval technique for nonobstructive azoospermia? A systematic review. Hum Reprod Update 2007; 13: 539-49.

39 Amer M, Haggar SE, Moustafa T, Abd El-Naser T, Zohdy W. Testicular sperm extraction: impact of testicular histology on outcome, number of biopsies to be performed and optimal time for repetition. Hum Reprod 1999; 14: 3030-4.

40 Raman JD, Schlegel PN. Testicular sperm extraction with intracytoplasmic sperm injection is successful for the treatment of nonobstructive azoospermia associated with cryptorchidism. J Urol 2003; 170: 1287-90

41 Chan PT, Palermo GD, Veeck LL, Rosenwaks Z, Schlegel PN. Testicular sperm extraction combined with intracytoplasmic sperm injection in the treatment of men with persistent azoospermia postchemotherapy. Cancer 2001; 92: 1632-7.

42 Palermo GD, Schlegel PN, Colombero LT, Zaninovic N, Moy F et al. Aggressive sperm immobilization prior to intracytoplasmic sperm injection with immature spermatozoa improves fertilization and pregnancy rates. Hum Reprod 1996; 11: 1023-9.

43 Schiff JD, Palermo GD, Veeck LL, Goldstein M, Rosenwaks Z et al. Success of testicular sperm extraction [corrected] and intracytoplasmic sperm injection in men with Klinefelter syndrome. J Clin Endocrinol Metabol 2005; 90: 6263-7.

44 Lin YM, Hsu CC, Wu MH, Lin JS. Successful testicular sperm extraction and paternity in an azoospermic man after bilateral postpubertal orchiopexy. Urology 2001; 57: 365.

45 Mininberg DT, Rodger JC, Bedford JM. Ultrastructural evidence of the onset of testicular pathological conditions in the cryptorchid human testis within the first year of life. J Urol 1982; 128: 782-4. 